\title{
Time for Ontology? The Role of Ontological Time in Anticipation
}

\author{
Tina Röck ${ }^{1}$ (D)
}

Received: 3 August 2016/ Accepted: 27 November 2017/Published online: 29 December 2017

(C) The Author(s) 2017. This article is an open access publication

\begin{abstract}
In this contribution, I will argue for an ontological understanding of time as temporality. This, however, implies that in a certain sense being is temporality, by which I mean that (1) on an ontological level temporality is nothing but the process of change, i.e. the dynamic aspect of being in its becoming, changing, and perishing, and (2) that concrete beings are not merely in time, but they are temporal. This leads to the conclusion that actual time is the process of change that becoming beings are, as well as the conclusion that reality is fundamentally temporal as argued by process metaphysicians like Alfred North Whitehead, Henri Bergson, Martin Heidegger, and Gilles Deleuze. The investigation begins with first establishing the methodological difficulties involved in thinking temporality as an ontological feature. In a second step, dynamic ontologies are introduced as the conceptual background best suited to think ontological temporality and their difference to event-ontologies is explained. Finally, the distinction between temporality and linear time is clarified. This introduction of temporality ends with some arguments for the existence of temporality that are inspired by Aristotle's famous investigations into the nature of time. After having thus introduced temporality as an ontological feature and argued for the existence and relevance of it, its implications for our understanding of the dimensions of time and especially for anticipation are discussed.
\end{abstract}

Keywords Temporal ontology $\cdot$ Process philosophy $\cdot$ Anticipation $\cdot$ Future $\cdot$ Past

Tina Röck

t.rock@dundee.ac.uk

1 University of Dundee, Room 3.9, Tower Extension, Nethergate, Dundee DD1 4HN, UK 


\section{To Be or Not to Be: The Existence of Time and of Ontological Temporality}

\subsection{Ontological Propaedeutic}

The intersection of ontology and time gives rise to two fundamental issues. One is the issue of defining the ontological status of time-of temporal becoming and temporal relations, i.e. the question of the nature of time as investigated by McTaggart or in positions like presentism, eternalism and the like. This issue will not be the main focus in what follows. The other issue is the question of the temporal status of existing entities. This is the question that will shape this contribution, even though the attempt to investigate the nature of temporality by looking at entities that are becoming and changing in time might sound rather unfamiliar and confusing to most philosophers.

One reason for this confusion is the fact that in order to understand time or ontology within this framework one has to look simultaneously at both to understand either one. This means that time cannot be understood without considering beings and beings cannot be understood independently from time or temporality. This position implies that being is temporality, by which I mean that (1) on an ontological level actual time is nothing but the dynamic process of becoming, being and perishing, and furthermore (2) that beings are fundamentally temporal. This approach renders any investigation into either matter rather complicated and is thus a highly unusual research path to propose. But I hope to be able to render these presuppositions less counter-intuitive in the course of my paper by arguing that strictly speaking temporality and dynamic being are just two aspects of one and the same process. We cannot separate the one from the other in our investigation of reality without gross oversimplification.

A further source for the aforementioned confusion might lie in the fact that traditionally ontology is often considered to be the study of fundamental entities that constitute reality and not a study of the temporal processes and events that bring forth beings. In phrasing the ontological project this way the general ontological question of what there is is automatically transformed into a question concerned with the fundamental building blocks of (generally physical) reality. This re-phrasing of the ontological project presupposes that the basic constituents of reality are well-defined and essentially unchanging entities or elements that make up what we call reality. This type of ontology is best characterized as a building-block-ontology.

Now, the building blocks making up reality might be considered temporally extended (as in event ontologies as the term is used in analytic philosophy ${ }^{1}$ ) or not temporally extended (like in many substance ontologies or trope ontologies). However, while substance and trope views generally consider time to be unrelated to the existing entities, event ontologies usually consider time to be a fundamental property of event-entities. But what unifies both varieties of building-blockontologies is that temporality as a quality, i.e. the flow of time that can be

\footnotetext{
1 In analytic philosophy this understanding of event ontology is very prominent and has for example been defended by Hacker (1982) or Cresswell (1986).
} 
experienced or the processual quality of time has no intrinsic relation to the entities that exist.

While, at first glance, one might conflate dynamic ontologies (i.e. all forms of process-ontology from Heraclitus to Whitehead) and event ontologies (i.e. the position that considers parts of the building blocks of reality to be temporally extended events like weddings, football games or lightening), there is a fundamental difference between these positions. In any truly dynamic ontology becoming has to be predicated from the beings themselves and not from the event-entities they are involved in. Furthermore events in event ontologies are well-defined entities, they have a fairly clear beginning and end. So events retain the building block structure, even if they are temporally extended building blocks. Therefore, even though temporality might seem to play a bigger role in event ontologies, they still should be considered a type of building-block-ontology and as such cannot take into account actual or existing time, i.e. temporality as a continuous interrelated flow of beings. Just a little side note to clarify the term 'event ontology', that might confuse some readers. I am aware that Whitehead, a dynamic philosopher par excellence, first conceptualised his dynamic ontology as an event ontology before devising an organic philosophy. While the Whiteheadian understanding of event ontology, i.e. events are ontologically basic processes, would fall under the category of dynamic ontology as it is used here, this is not the type of event ontology I am referring to. I am using the term as it is used in analytic philosophy in referring to temporally extended events like accomplishments, activities achievements or states that beings can be involved in. (compare Faye et al. 2001 or Bott 2010).

There are, of course, events in dynamic ontologies as well, but these events have temporality only thanks to the becoming beings involved in these events. In any truly dynamic ontology not the temporally extended event-entities are ontologically fundamental but the moving and changing temporal beings themselves. While in all types of building-block-ontologies existence is usually considered to be more or less independent from the flow of temporality and the corresponding ontological changes in beings, this is not the case in dynamic ontologies.

An analogy might help to clarify this point. Temporally extended event-entities are well-defined blocks, with a precise beginning and a precise end that can exist somewhat independently from all other events. However, in dynamic ontologies all beings are fundamentally related to each other in their respective processual being, their becoming, evolving, and perishing. The difference is analogous to the difference between a Lego-brick house and patches of watercolour. One can extract a single brick from a Lego-house without too much hassle and generally without influencing the structural integrity of the whole. Considering a watercolour painting things are different. After the watercolours blend together, as they are supposed to do, there are only vague borders between the colours, so that there is no exact or simple way to separate one colour from the other. This interconnection between the various colours is enhanced by the fact that the colours continue to blend further as time passes (as long as there is enough water). I hope that the analogy of the watercolour painting sheds light on the way temporal beings are fundamentally constituted by the various ways they interact through time, so that it is impossible to separate one being from the rest without oversimplification. 
Also living beings are another excellent example for this interconnectedness and interactivity. One cannot separate me from all the processes influencing and constituting my being - the air I breathe, the metabolic processes changing the food and drink I consume in order to preserve my flesh, the books and conversations that shape my thoughts-without abstracting from what I am as a concrete being and turning me into just one more instance of a general - though timeless and objective entity - woman or, even more general, a human being.

Dynamic ontologies are unified by the claim that the quality of flow in lived time, i.e. temporality, cannot be divorced from being without oversimplification. In a dynamic understanding of reality thus temporality becomes a genuine ontological feature; there is no ontological 'basis' or 'substrate' that is not fundamentally temporal. In this ontology change or being temporal is not an accident but the primary property of what there is - a property so fundamental that it takes the role 'existence' plays in building-block-ontologies. There are no a-temporal ideal entities shaping the true form of existence behind the changing world of our perception. In dynamic ontologies reality is a constant flux, a process whose future is not yet decided and whose past is continuously formed by the present. This openness of the past as well as the future is one of the most fundamental differences between building-block-ontologies and dynamic ontologies and the reason dynamic ontologies are so apt at better anticipating the future.

When talking about the temporality or the flow of time, the one distinction most philosophers will think of is McTaggart's distinction between the A-series of time, representing time as past, present, future and the B-series of relational time (McTaggart 1908). But there is a fundamental difference between the flow of time described by McTaggart's A-series and the ontological quality described by the term 'temporality' or 'concrete temporality' as it is used here. McTaggart's A-series description reduces the flow of time to a relational ordering as to a before and after of events as described in the B-series plus the quality of change. Neither series, nor their combination can account for the quality of flow or continuous change that can be experienced or temporality itself. Looking at the A-series, it allows for change, but only concerning the fact that some event first has the quality of being in the future, then in the present and finally it will have the property of being in the past. However, the focus in characterising time through this series rests on the fact that events pass through points on the timeline or are those points on the timeline. What changes or flows is neither time nor temporality, any change considered is in correlation to the tense-quality an event has at any given time. Since the flow of concrete temporality does not happen in the future or the past, it is not quite clear how tensing events is supposed to help in explaining the actual flow given in experience. It is, of course, possible to extrapolate far into the future or into the past from the present, however, what is present now is a temporally extended present. ${ }^{2}$

\footnotetext{
${ }^{2}$ Of course we could look at this present flow and with Husserl identify its protentional and retentional dimensions, its experiential roots in the past and its projections into the future. Nonetheless these aspects, strictly speaking, are neither future nor past, they are present and that is all that is given in direct and immediate experience. To access the past we need the aid of memory, for the future we need speculation or projection, processes which mix immediate experience with conceptual or theoretical engagement.
} 
Both series thus are examples of the way we generally conceptualise time, namely in analogy to space. Both are founded on our understanding of space, leading to the intuition of a timeline on which all events are ordered, whether that timeline is considered to possess the properties of McTaggart's A-series or B-series is largely irrelevant for this discussion. McTaggart presented a concept of time that cannot provide a sufficient basis for understanding the kind of enduring temporality that we experience and that is implied by dynamic ontologies, both his series (and this goes for the $\mathrm{C}$-series as well) look at a succession of events on a timeline. Even if his A-series allows for change, its rendering reduces duration and change to a change in the quality of being-past, being-present or being-future and thus also eliminates what Bergson calls 'real time':

For [...] [something] which does not change does not endure and a [...] state which remains the same so long as it is not replaced by the following state does not endure either. Vain, therefore, is the attempt to range such states beside each other [...]: never can these solids strung upon a solid make up that duration which flows. What we actually obtain in this way is an artificial imitation [...], a static equivalent which will lend itself better to the requirements of logic and language, just because we have eliminated from it the element of real time. (Bergson 1922, 4)

\subsection{Spatialized Time as a Concrete Example of a Time Without Temporality: Henri Bergson}

But why can neither of McTaggart's renderings of time cope with ontological temporality? This is the case because both these definitions of time, namely time as the sum of past, present and future as well as time as an ordered before and after, are ways of understanding time as 'spatialized', i.e. they understand time in analogy to space. So both McTaggart's A- and B-series are just two different ways of describing 'spatialized' time. With Bergson I will take a closer look at this very common version of a concept of time without temporality. Then I will contrast it with a general description of a non-spatial understanding of time as temporality, along the lines of Bergson's durée.

'Spatialized' time according to Bergson can be defined as a pure extension between an infinite past and an infinitely distant future. The past and the future exist on a timeline and any point in time can be situated on this line, creating a relative past and relative future (or a relative before and a relative after) depending on its placement. Any length of this timeline makes up a temporal extension and these extensions too can be correlated as to their respective position on the timeline. There are no two contemporary points in time just as there is no instant before another instant:

To speak thus is to ignore the cardinal difference between concrete time, along which a real system develops, and that abstract time which enters into our speculations on artificial systems. What does it mean, to say that the state of an artificial system depends on what it was at the moment immediately before? There is no instant immediately before another instant; there could not be, any 
more than there could be one mathematical point touching another. (Bergson 1922, 22)

The position of the event on the timeline or its relation to other time-points within the time-container provides temporal indices for the events that happen, it marks them, but it is not integral to them. In a certain sense 'this time' and its 'now' as well as 'before' and 'after' are transcendent to the events taking place in time. This understanding of time is a concept of time without temporality and it is the understanding of time successfully used in the sciences.

For the moment, we will confine ourselves to pointing out that the abstract time attributed by science to a material object or to an isolated system consists only in a certain number of simultaneities or more generally of correspondences, and that this number remains the same, whatever be the nature of the intervals between the correspondences. [...] Common sense, which is occupied with detached objects, and also science, which considers isolated systems, are concerned only with the ends of the intervals and not with the intervals themselves. Therefore the flow of time might assume an infinite rapidity, the entire past, present, and future of material objects or of isolated systems might be spread out all at once in space, without there being anything to change either in the formulae of the scientist or even in the language of common sense. The number $t$ would always stand for the same thing; it would still count the same number of correspondences between the states of the objects or systems and the points of the line, ready drawn, which would be then the "course of time". (Bergson 1922, 9)

So this spatial understanding of time is insensitive to a speeding up or slowing down of the flow of time. Whether time flows as it does, whether it flowed faster or slower would not make a difference to this understanding of time, since it is an abstract and generalised view. In contrast, a change in the speed of the flow of time would make a huge difference for us living beings. Consequently the abstract understanding of time cannot account for certain aspects of lived time and vice versa, so the two understandings of time should not be conflated.

In the abstract and spatial understanding of time the 'now' is a privileged point in time, even though it is mostly considered to be merely the ideal point of contact, the point in time where the future transforms into the past. Just like the experience of the passage of time, this 'now' is often considered to be merely the result of a living consciousness looking at the moments of time on the timeline too. This 'spatialized' time of succession (be it 'objective' succession or 'relational' succession) is independent from the events or changes that happen during the time investigated, just as space is independent from the objects that take up space. In analogy to the concept of a space-container in which extended things exist, 'spatialized' time is often conceptualised as a time-container in which events and things can happen. Time itself is considered to be exterior to these ongoing events.

There are many problems connected to this prevalent understanding of time, leading many philosophers to conclude that the flow of time does not exist at all (or that some dimension of time does not exist), even if change, flow, and temporality 
are everyday experiences. But what would happen if we distinguished spatial time as a concept from the quality of temporality as we experience it and instead of explaining our experience of time through an abstract and inadequately simplified concept used a qualitative concept of time that takes into consideration the experience of flow?

\subsection{Time and Ontology: A Fundamentally Aristotelian Issue?}

Aristotle famously introduces the problem of the relation between time and

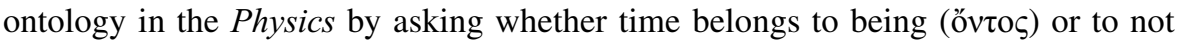

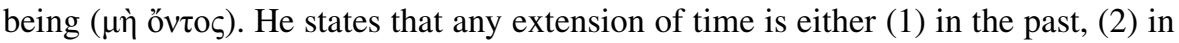
the future or (3) connects past and future (the moment of now). But, he argues further, the past does not exist anymore and the future does not yet exist, so neither of them can be genuinely real. Finally the moment of 'now' is nothing but the everchanging point dividing the past from the future and thus cannot be said to exist independently from past and future. (Phys. 217b29-218a30) Aristotle looks at the nature of this 'now' again and again in these passages, with different results. (for example Phys. 218a3seq) But he ends chapter 11 with the claim that 'now' is not time, but an attribute of time. (Phys. 220a21) So if all of the components that constitute time do not exist independently (now) or simply do not exist (past, future), we have to conclude that the sum of these components including the attribute 'now' does not exist either. (Phys. 217b33-218b30)

But this is not the only characterization of the nature of time in these passages of the Physics. "[I]n Aristotle's analytic [...] time turns out not to be a being qua itself but an attribute of motion, an interval." (Harry 2015, 33) Aristotle argues that in our perception time and movement always occur together and that there is no time without a marked motion. Time can therefore also be considered to be the measure of movement in respect to the before and after. He clarifies this correlation by explaining that movement and the passing of time are closely related, but that neither can be reduced to the other since time is not the same as movement but it belongs to movement ${ }^{3}$ (as its measure). He concludes that we do not only measure movement through time, but we also measure time through movement or change. (Phys. 220b14-16)

One might conclude from these somewhat cryptic passages in Aristotle that abstract time considered as the sum of past, present and future cannot be said to exist in itself, but that time as the measure of motion is somehow more closely related to (changing) being and thus has a stronger connection to existence. It might still not exist as a substance or a separate entity, but there is something about it relating it to being and its movements. Beginning with Aristotle's arguments one can speculate as to the difference between abstract time (without temporality) and time understood as concrete temporal flow (or temporality): while the static time of past, present and future is not part of being (as proven by the first argument), the

\footnotetext{
3 Time is not identical to change or movement, since (1) change presupposes movement in space and time has no location in space - time is not spatial — and (2) change involves different speeds, changes can become faster or slower, time cannot be described this way. So time cannot be identical to change or movement. (218b18) But time belongs to movement since the experience of time necessarily implies motion and change.
} 
flow of time which can be used to measure change (implied by the second argument) has a connection to being. Incidentally there is an often overlooked distinction between these two times that Aristotle proposes in 218a1. Here Aristotle distinguishes infinite time (ho apeiros chónos)—which I read as time without temporality-from (concrete) taken time (ho aei lambanómenos chónos), which I understand as time as we take/experience it, i.e. temporality.

But I would like to go even further and claim that if temporality is considered a quality realized or instantiated by movement and change, i.e. by the process of becoming and changing beings, it is just as real as the becoming beings themselves. This unorthodox reading of the Aristotelian arguments gives the flow of time an ontological twist resulting in an understanding of temporality that is entangled with movement and change. But I am not the only one making this connection: Harry, for example, argues that time's "actual existence depends on the motions of natural beings; it is not an a priori or self-subsistent being" (Harry 2015, 33). This reading opens up a new perspective on time, the same perspective that follows naturally from any dynamic understanding of reality, namely time as the quality of change, of flow generated by the becoming of beings. Time as temporality is the concrete flow that fundamentally shapes our everyday experience of an unfolding world. It is this flow that we refer to when we talk about our experience of time.

We find the same dichotomy between our everyday experience of the passing of time and the abstract understanding of time without temporality at the root of Augustine's famous quote: What then is time? If no one asks me, I know what it is. If I wish to explain it to him who asks, I do not know.-There is something fundamental and obvious about our experience of temporality, but as soon as we try to conceptualise or explain it in terms of abstract concepts, we get caught in endless contradictions, which have led many philosophers to deny the actual existence of the flow of time, considering it a mere figment of our imagination or consciousness.

Even if the abstract understanding of time is a highly useful tool, a fact which I would not want to dispute, in certain regards it does not describe concrete reality precisely enough and thus leads us astray when we try to actually understand the concrete world we live in or when we attempt to anticipate concrete future developments. ${ }^{4}$ But what would happen if we distinguished the abstract understanding of time without temporality from the quality of temporality that we experience and if, instead, we used the qualitative concept of temporality where appropriate?

\section{The Quality of Concrete Temporality: Fundamental Issues}

This proposed move from time to temporality leads to many conceptual and practical changes in our understanding of concrete time. I will begin with the most fundamental question: if only the quality of temporality and its corresponding ontological aspect — changing or moving beings — do exist, what does that mean for the dimensions of past and future? Is time as the temporality of changing beings

\footnotetext{
${ }^{4}$ See for example the new book by Physicist Lee Smolin Time Reborn (Smolin 2014).
} 
necessarily reduced to the present, the actual change in progress or can it also provide a basis for the dimensions of past and future, dimensions as much part of our experience as current temporality?

\subsection{Concrete Temporality and the Past}

The simple answer is: can we even imagine a process or change that is restricted to the present moment? Processes are necessarily temporally extended. Any current change has its roots in the past and its possible results stretch far into the future.

Furthermore it seems rather adequate to consider the present not merely as a privileged starting point of our experience but instead as a result of past processes, even if temporality is rarely approached this way: what happens now could not be happening without the past events that have led up to it. This way of looking at temporality discloses the past as being virtually present in every moment of 'now' as its sine qua non. The easiest way to understand this correlation is to investigate living beings. The process of existence of a plant is one continuous process, where each stage is a development and growth based on and coming from its antecedent stage: the past, the seed, its germination, its growth and development, and the generation of a bud that then will open into a flower are all moments of one continuous process. The bloom present now could not exist independently from the past developments leading up to it. There is no bloom without the seed and it would not make sense to conceive of the flower as independent from the seed. In a certain sense the seed is still present in the flower. The seed and its 'experiences', i.e. the way it germinated, the nutrients present in the soil at that time, the way the light fell on the offshoot and so forth-all these concrete and contingent factors in the past influence(d) the development of the plant, change(d) and forme(d) it and gave rise to what is present now. Any contingent and concrete past influence is integrated in the development and is now virtually present in the actual bloom. The present is the embodied result of past developments, since it is this past that results in the present and that is still (virtually) present now. The same correlation can also be expressed from the point of view of the present: the present re-instantiates the past by adding something to it, by developing and growing it further in its unfolding towards a future. While a dynamic understanding of time is thus fully temporally extended (without any lean boarders or limits), most static conceptions of time are organized around the present, as if the present was the truly existing basis from which past and future have to be deduced. Keeping the plant metaphor in mind it becomes evident that such a preference of the present over the past is only possible if time is divorced from ontological existence. The temporal unfolding existence of beings, however, is always and necessarily rooted in the past and directed towards the future. On this view reality grows and unfolds from the past through the present into the future.

But there is a further dimension shaping the interrelation of past and present. Bergson uses the concept of 'memory' to account for this interrelation. For Bergson the past is 'remembered' in the cumulative progression of duration. This remembrance is not a form of passive registration, but rather takes the form of an addition. With every new present that is added and thus integrated to the past, the past as a whole changes. The movement of duration or temporality is thus not a 
linear progression, since with every passing moment there is not only a change in the present but also a resulting change of the past as a whole. Every present moment that passes is a moment that is 'added to' the past and so the past grows along with and through the developments of the present. So, in a certain sense, the past as a whole is constantly changing due to a constant addition of present moments. The past is thus co-present with the present and the present gives rise to the past. Every present re-enacts its past and by re-enacting it transforms it. This temporal extension opens up room for creativity and novelty. In every present re-enactment or representation of the past there is room for creativity and newness, new experiences, new influences, and new forms of engaging with the virtual past or of anticipating the future. Any one of these processes can give rise to new instantiations that incorporate aspects that were not fully dispositioned by the past-they lead to open futures. This leads to the following characterization of ontological temporality: in ontologically embodied time (i.e. in temporality) events cannot be considered to actually move from the future to the present to the past. On the contrary, all existing beings move and evolve from the past through the present, from the present into the past and the future. And their sheer temporal existence incorporates, entwines and connects all of these dimensions in their constant becoming.

\subsection{The Rhizomatic Structure of Concrete Temporality}

This leads me to a second central aspect of ontological time: it is fundamentally relational on two levels. This is the case since (1) ontological time is the temporal extension of concrete beings that stand in relations to each other and (2) the temporal dimensions of past, present and future are themselves interrelated. They cannot be considered to be independent dimensions, just as the present being cannot be considered to be independent from its becoming in the past and its direction toward the future.

1. Since temporality in this understanding is so closely interrelated with becoming and the singular changing ontological beings engaged in the process of becoming, we have to contend with a multiplicity of 'temporalities', since every being in its becoming generates its own temporality. But all these temporalities coexist and intersect, thus creating a sort of temporal tapestry in which every single temporal becoming is interwoven with the other becomings. In order to create a common temporality that we are able to measure independently from the interrelation of becoming beings, i.e. abstract time, we pick one of these temporalities, be it the becoming being of a certain star in its relation to the earth or the time it takes caesium atoms to undergo a specific quantum transition, and use it as a standard-temporality that all other temporalities are measured against. This process helps us understanding the transition from experienced time as temporality to 'objective' time by using one temporality as the unchanging standard for all. The 'objectivity' of this procedure is grounded in the fact that the interval marked by these specific quantum transitions provides us with the most regular and reliable oscillator available to date. Time thus is not the measure of change anymore but the measure only of the change 
undergone in quantum transitions, which is the movement that is then used to measure all other changes.

If in our reflection on concrete reality (but not in science obviously) we wanted to take the plurality of becoming into account, we could not stipulate a single time. Time as ontological temporality is inherently plural and interrelated, so there are as many temporalities as there are becoming entities that are all connected into a tapestry of temporality that we can reflect upon, but not measure. Analogously there is an according multiplicity of 'pasts' that make up what we generally call 'the past' and just such a multiplicity of presents and virtual futures. The understanding of temporality in dynamic ontologies is therefore always multiple, facetted and interwoven and not linear, progressive or unified.

2. Over time many philosophers have claimed that neither the past nor the future do exist, since the one is not real anymore and the other is not yet real. As I argued in the preceding section in a dynamic ontology the past and the future are usually thought of as coexisting with the present in the present as its virtual dimensions. Only the interconnection of all three-past, present and future-allows for existence and temporality. Any process is a unity of past, present and future.

There have been several attempts to conceptualize this temporal extension of reality by focusing on the connection of past, present and future in various ways: one example is Husserl's description of phenomenological perception where Protention, Urimpression and Retention are inseparably intertwined in every moment of experience (see Husserl 1990). Heidegger ${ }^{5}$ utilizes a similar structure in Being and Time as well as in his later work to formulate a fundamental and fundamentally temporal ontology. In Whitehead the past and the aim towards the future are fundamental in shaping any becoming being (i.e. actual occasion) (see Whitehead 1978). The becoming being reflects its past and interprets it in light of a future aim. So that the temporal dimensions fundamentally shape the present becoming. Another example can be found in Bergson's and Deleuze's idea of virtuality, where past and future are considered ontologically connected, i.e. virtually present (or anwesend in Heidegger's sense) in the present moment. These thinkers share the conviction that the quality of temporality that merges past, present and future has to be taken seriously when trying to understand and describe concrete (or phenomenologically given) reality.

\section{Temporal Dimensions in Dynamic Ontologies and Anticipation}

\subsection{The Future}

While most static ontologies limit our possible engagement with the future at least to some extent, in a dynamic ontology we are granted and burdened with the

\footnotetext{
${ }^{5}$ Schon-sein-in-der-Welt: Gewesenheit, Sein-bei (dem momentan zu Besorgenden): Gegenwart, Sichvorweg-sein (im Entwurf): Zukunft (see Heidegger 1962).
} 
constant possibility to engage with the momentary processes in order to influence the future. The fact that in our everyday lives we constantly engage in anticipatory behaviour and that we try to influence the outcome of current developments shows that in regards to the future people generally appear to endorse a rather dynamic understanding of reality. We set ourselves goals and then try to engage with the present in such a way as to facilitate the manifestation of these goals. If I want to become a doctor, I will have to study now, if I want to harvest tomatoes, I should plant the seeds at an appropriate time and so on. In static ontologies these behaviours are usually explained as bringing forth what has been present in the modus of dynamics (potential) all along. Engaging with the present is not to be understood as bringing forth a new future, but as an unveiling of what has been there all along as a disposition or an as of yet unexpressed potential, for example in the shape of a pre-existent essence of beings (esp. in Aristotelian ontologies). What is and what will be is pre-determined, at least to a large extent. There is not much room for creativity, novelty or freedom. Still our everyday life is infused with the feeling of freedom and the (admittedly subjective) experience of having the power to shape the present in order to influence a future outcome.

So if we want to take the project of anticipation seriously, we will have to adopt a dynamic ontology, since it is the kind of ontology able to systematically find active and creative (and non futile) engagement with the future through acting on the present. And a dynamic understanding of reality, if it describes the way concrete reality is structured, is anticipatory by nature. The fact that we constantly engage in anticipatory behaviour and that we generally try to influence the outcome of current developments shows that in regards to the future we are decidedly tending toward a dynamic understanding of reality. In regards to the past, on the other hand, we appear to have decidedly more static preconceptions. This is why in what follows I will argue that even the past, as envisioned by dynamic ontologies, has an anticipatory dimension.

\subsection{The Past}

The aim of this last section of the paper is to show that dynamic ontologies imply an anticipatory dimension also in their understanding of the past, since in a dynamic understanding of reality the past is not cleanly cut off from the present or the future but is in constant engagement with both.

In most building-block-ontologies and in most proposed theories of time the past is imagined either as a static repository of all that has happened or it is simply considered to be irrelevant since it does not exist anymore. But if we take the dynamic and relational structure of reality seriously, we have to consider the idea that the past is not a static repository, but that it is just as dynamic and evolving as the present. If reality is a constant flux, a truly open process, then the past cannot be considered an unchanging block or static repository. It has to be understood as changing and evolving with the present, since it is continuously formed, folded and expanded by the becoming present that becomes part of the past as reality unfolds. This leads to the conclusion that by actively attempting to shape the present in order to influence the future we are simultaneously also able to influence the past as a 
whole. This, of course, does not mean that we can just simply change specific events that happened in the past (even if in exceptional circumstances even that might be possible), but that by changing the past as a whole we can influence the role these events play(ed) and thus influence the effect they have on the present and the future.

It is also the case that we look to the past in order to decide how to anticipate best. More often than not the past is referred to in order to promote change in policy concerned with the future or to argue in favour of keeping things the way they 'always were'. Phrases like 'this has worked before', or 'why fix what is not broken' and the well-known 'it did not work before, why should it work next time?' are just some examples of slogans concerned with future outcomes by relying on past experiences. Used this way the past again appears to be something akin to an objective repository of information that can be used to give anticipation an objective and indubitable fundament. From the perspective of a dynamic world-view things are not that simple. There are two fundamental problems with this treatment of the past as a guide for anticipation from the point of view of a dynamic understanding of reality:

1. There is no singular absolute account which is able to unify the fractured and evolving multitude we usually call the past.

2. Every past event and any historic moment was set in very specific circumstances. Every moment in history is radically singular and not repeatable. So simply applying to the present or the future what has worked in the past is often inadequate.

In the last part of this paper I will take a closer look at these problems.

1. There is no singular absolute account which is able to unify the fractured and evolving multitude we usually call the past.

The past is co-present with the present, since every present realizes its past and transforms it by re-enacting or re-instantiating it. With Bergson we could describe this process by saying that the past is 'remembered' in the cumulative progression of duration when describing the fact that the past is always virtually present. But the important point is that with every new present that is added and thus integrated (in)to the past, the past as a whole changes. So in a certain sense the past is constantly changing due to a constant addition of present moments. So if the present can influence and shape the past by becoming integrated into the past, then in our present actions we are not only responsible for the present but, at least to a certain degree, also for the past (as it will appear for the future). The present cannot be absolved from its responsibility towards the past. Furthermore the past cannot absolve us from any harm that we presently commit - there is just no single legitimate or unchanging description of the past that could justify any present harm, domination, disqualification and the like. Consequently there is also no absolute basis in the past to be able to claim a superior status for any culture, race or achievement or to be able to determine a superior form of living.

The past cannot guide our present actions or our anticipation in a reflected and straightforward way as it is often done by using expressions like 'it did not work 
before, why should it work next time?', not only because our present actions continuously contribute to and thus change the past as a whole, but also because:

2. Every past event and any historic moment was set in very specific circumstances. It is radically singular and not repeatable. So that strictly speaking a simple learning from the past is impossible.

So does that mean that we cannot learn from the past at all? No, it is just not as simple as it is usually made out to be. We cannot merely draw on similarities between past and present or future situations, but we also have to see the specific differences for an adequate anticipation. Therefore we cannot just compare the acts of violence currently committed against refugees or by refugees with other instances of violence, but we also have to look at the aspects that make the present situation unique and different to be able to take anticipatory action adequate to our situation and not merely adequate to a situation long past.

So we cannot simply depart from the premise that the hatred against foreigners or committed by foreigners now is the same as it was in some other moment of time, instead we need to ask the much more subtle question of HOW is the hatred the same as before and HOW it differs in order to take action adequate to the present situation and not merely adequate to a situation long past. This is to say that we have to take into account the intensities and qualities of a situation and should not rely on 'objective' facts alone as a basis for our anticipatory strategies.

\section{Conclusion}

The aim of this paper was to show that any dynamic ontology, from Heraclitus to Bergson, from Whitehead to Deleuze is anticipatory in nature for structural reasons. But this anticipatory dimension does not only hold in regards to the future, which is quite intuitive, but also in regards to the understanding of the past. This outcome might sound quite surprising, but I hope to have shown that it follows quite naturally from a dynamic understanding of reality.

If we now ask what consequences this understanding of reality as genuinely temporal has for the role that philosophy can play in anticipatory behaviour, provided this understanding is accurate, then I would claim that the best way for philosophy to contribute to anticipation is to aid in developing an adequate mind set allowing for anticipation in a temporal world. The central term here is adequate. Any action in a world that is genuinely temporal, whose past has anticipatory moments, where time and being cannot be separated and where there is room for genuine freedom and creativity cannot be based on an a-temporal and ideal fundamentum inconcussum, be it logical, linguistic or ontological. In a truly dynamic world there is no indubitable fundament on which we could build a necessary philosophical system adequate to guide our engagement with the world. A truly anticipatory philosophy has to come to terms with the dynamic and vague 
nature of actual reality instead of devising an ideal world as a guide to desirable behaviour and outcome, as so many philosophers have done before.

The dynamic nature of reality thus might provide the reason why philosophy, as long as it is used as a guide to ultimate truths and answers, fails to provide us with a good guide to a better future. What then if we attempted to use philosophy as a tool to find answers adequate to the actual qualitative temporality instead of looking for ideal and absolute answers?

Open Access This article is distributed under the terms of the Creative Commons AttributionNonCommercial 4.0 International License (http://creativecommons.org/licenses/by-nc/4.0/), which permits any noncommercial use, distribution, and reproduction in any medium, provided you give appropriate credit to the original author(s) and the source, provide a link to the Creative Commons license, and indicate if changes were made.

\section{References}

Aristotle (1984) In: Barnes J (ed) Revised oxford translation (ROT) of the complete works of Aristotle, vol 2. Princeton University Press, Princeton

Bergson H (1922) Creative evolution. An alternate explanation for Darwin's mechanism of evolution (trans: Arthur Mitchel). Reprint of the 1911 Edition. MacMillan and Co, London

Bott O (2010) The processing of events. John Benjamins, Amsterdam

Cresswell MJ (1986) Why objects exist but events occur. Stud Logica 45:371-375. Reprinted in Events, pp 449-453

Faye J, Urchs M, Scheffler U (eds) (2001) Things, facts and events. Rodopi, Amsterdam

Hacker PMS (1982) Events, ontology and grammar'. Philosophy 57:477-486. Reprinted in Events, pp 79-88

Harry CC (2015) Chronos in Aristotle's physics. On the nature of time. Springer, London

Heidegger M (1962) Being and time (trans: J. Macquarrie and E. Robinson). Basil Blackwell, Oxford

Husserl E (1990) On the phenomenology of the consciousness of internal time (1893-1917) (trans: J. B. Brough). Kluwer, Dordrecht

McTaggart J (1908) The unreality of time. In: Mind. A quarterly review of psychology and philosophy, vol 17/1908, pp 457-474

Smolin L (2014) Time reborn: from the crisis in physics to the future of the Universe. Penguin, London Whitehead AN (1978) Process and reality. An essay in cosmology. In: Griffin DR, Sherburne D (eds) Corrected edition. The Free Press, New York 